\title{
THE BLIND INFECTED WITH LEPROSY IN JAPAN
}

It is calculated that there are about 10,000 patients with leprosy in 15 leprosaria in Japan, and that of these about 1,000 are blind. However, both the number of those with leprosy and the proportion of those who are blind are diminishing. The leprous blind are doubly unfortunate, as in addition to being unable to read ordinary type, the anaesthesia of their fingers makes it impossible to read braille. However, a way out has been found by which they are able to read braille with their tongues or lips. Already (April, I955) there are Io8 braille typists, 45 tongue readers and 5 lip-readers. Also 73 are able to read braille with their fingers. This new movement has brought a fresh incentive to live to many of the patients. There is reported to be more blindness among those with leprosy in the north of Japan than in the south, and this is considered to be because the greater cold in the north encourages the more severe (lepromatous) form of leprosy, the form which causes blindness. Two reasons are given for the fact that blindness among those with leprosy is diminishing: (I) treatment with Promin, and (2) many of those already blind perished in the war. 